\title{
Dietary composition and its associations with insulin sensitivity and insulin secretion in youth
}

\author{
Mélanie Henderson ${ }^{1,2 *}$, Andrea Benedetti ${ }^{1,3}$ and Katherine Gray-Donald ${ }^{4}$ \\ ${ }^{1}$ Department of Epidemiology, Biostatistics and Occupational Health, McGill University, Montreal, QC, Canada \\ ${ }^{2}$ Division of Endocrinology, Department of Pediatrics, Centre Hospitalier Universitaire Sainte-Justine, \\ Université de Montréal, 3175 Chemin Côte Ste-Catherine, Montreal, QC, Canada H3T 1C5 \\ ${ }^{3}$ Department of Medicine, McGill University, Montreal, QC, Canada \\ ${ }^{4}$ School of Dietetics and Human Nutrition, McGill University, Montreal, QC, Canada \\ (Submitted 4 December 2012 - Final revision received 4 July 2013 - Accepted 8 July 2013 - First published online 19 September 2013)
}

\section{Abstract}

The objectives of the present study were to examine the associations between macronutrient intake and insulin sensitivity (IS) and insulin secretion (ISct), taking into consideration moderate-to-vigorous physical activity (MVPA), fitness and sedentary behaviour. Caucasian youth ( $n$ 630) aged $8-10$ years at recruitment, with at least one obese biological parent, were studied (QUebec Adipose and Lifestyle InvesTigation in Youth cohort). IS was measured using the homeostasis model assessment (HOMA) of insulin resistance and Matsuda IS index. ISct was measured using HOMA $2 \%-\beta$, the ratio of the AUC of insulin:glucose over the first $30 \mathrm{~min}$ (AUC I/G $\mathrm{G}_{t=30 \mathrm{~min}}$ ) of the oral glucose tolerance test and AUC I/ $\mathrm{G}_{t=120}$ min over $2 \mathrm{~h}$. Fitness was measured using $\mathrm{VO}_{2 \text { peak }}$, percentage of fat mass by dual-energy X-ray absorptiometry, and $7 \mathrm{~d}$ MVPA using accelerometry; screen time (ST) by average daily hours of self-reported television, video game or computer use. Dietary composition was measured using three non-consecutive dietary recalls. Non-parametric smoothing splines were used to model nonlinear associations; all models were adjusted for age, sex, season, pubertal stage, MVPA, fitness, ST and adiposity. The percentage of total daily energy from dietary protein, fat, saturated fat and carbohydrate and the consumption of dietary vitamin D, sugar-sweetened beverages, fibre and portions of fruits and vegetables were taken into consideration. No dietary component was associated with any measure of IS after adjusting for MVPA, fitness, ST and adiposity. For every $1 \%$ increase in daily protein intake (\%), AUC I/G $\mathrm{G}_{t=30 \mathrm{~min}}$ decreased by $1 \cdot 1 \%(P=0 \cdot 033)$. Otherwise, dietary composition was not associated with ISct. While long-term excess of energy intake has been shown to lead to overweight and obesity, dietary macronutrient composition is not independently correlated with IS or ISct in youth.

\section{Key words: Fat: Carbohydrates: Protein: Adiposity: Adolescent/children: Insulin secretion: Insulin sensitivity}

The prevalence of childhood obesity has increased exponentially over the last three decades, having almost tripled in Canadian youth ${ }^{(1)}$. There is growing evidence to suggest that impaired fasting glucose (IFG) and impaired glucose tolerance (IGT) are also on the rise in North American children and adolescents ${ }^{(2)}$. Furthermore, the prevalence of these pre-diabetic states is particularly high in obese youth ${ }^{(3,4)}$. The high prevalence of abnormal glucose homeostasis in this population suggests that IFG and IGT probably represent a significant health burden in Canadian youth.

There is extensive evidence showing that lifestyle modifications prevent or delay the transition from IFG/IGT to type 2 diabetes (T2D) mellitus in adults. In the Diabetes Prevention
Program study, intensive lifestyle interventions that included physical activity and dietary modifications decreased the rate of progression from abnormal glucose homeostasis to frank T2D by $58 \%{ }^{(5)}$. Studies on the association between dietary macronutrients and insulin sensitivity (IS) and insulin secretion in youth, however, is sparse and largely inconclusive. Lindquist et $a l^{(6)}$ found that dietary fat was not independently associated with IS or insulin secretion in ninety-five pre-pubertal/early pubertal African-American and white children. Conversely, Arslanian and colleagues found that a higher fat:carbohydrate ratio was associated with lower IS in forty-four pre-pubertal children (twenty-two African-American and twenty-two white $)^{(7)}$. These studies

Abbreviations: HOMA-IR, homeostasis model assessment of insulin resistance; IFG, impaired fasting glucose; IGT, impaired glucose tolerance; IS, insulin sensitivity; Matsuda ISI, Matsuda insulin sensitivity index; MVPA, moderate-to-vigorous physical activity; OGTT, oral glucose tolerance test; QUALITY, QUebec Adipose and Lifestyle InvesTigation in Youth; SSB, sugar-sweetened beverages; ST, screen time; T2D, type 2 diabetes.

*Corresponding author: M. Henderson, fax +1 514345 4988, email melanie.henderson.hsj@gmail.com 
focused on relatively small samples of children and did not account for other lifestyle habits that might act as confounders.

Similarly, the limited data considering protein intake and insulin dynamics in childhood have led to conflicting conclusions. Two studies on milk consumption in relation to IS and insulin secretion have drawn opposite conclusions concerning their association ${ }^{(8,9)}$. Hoppe et al. $^{(8)}$ also found that the addition of meat to children's diet led to unchanged IS and increased $\beta$-cell function. These studies, however, did not account for total protein intake, considered exposure over a short time frame and were conducted on relatively small sample sizes.

In the same way, data obtained from studies examining associations between dietary carbohydrate and insulin dynamics in youth are conflicting, and these studies assessed the acute effects of these diets in randomised trials on very small sample sizes, with exposure being over a short period of time (maximum 12 weeks). Sunehag et al. ${ }^{(10)}$ examined the effect of high-carbohydrate/low-fat diets in a sample of thirty-six healthy, non-obese pre-pubertal and adolescent youth for a $7 \mathrm{~d}$ period. They found that in these children, high-carbohydrate diets were not associated with any changes in IS in the pre-pubertal youth, but associated with improved IS in the adolescent group. Furthermore, pancreatic $\beta$-cell insulin secretion remained unchanged in both the pubertal and pre-pubertal youth. Davis et al. ${ }^{(11)}$ examined the association between carbohydrate and adiposity and insulin dynamics in a cohort of 120 obese Latino youth (mean age $14 \cdot 1$ years) with a family history of T2D. They found that total dietary sugar intake was inversely related to IS and insulin secretion, independent of sex, sexual maturation, total energy intake, body composition and non-carbohydrate macronutrient intake.

Studies examining chronic dietary exposures, in large numbers of youth, taking into consideration other lifestyle habits are lacking. The objectives of the present study were to determine the associations of macronutrient intake with insulin and glucose dynamics in children, taking into consideration physical activity, fitness and sedentary behaviour. Understanding dietary influences on glucose homeostasis will be useful for clinical practice and public health management if they are studied within the context of overall lifestyle habits and how these habits interact.

\section{Methods}

Participants of the QUebec Adipose and Lifestyle InvesTigation in Youth (QUALITY) cohort were studied using the baseline evaluation for the present study ${ }^{(12)}$. QUALITY is an ongoing longitudinal study of Caucasian youth aged 8-10 years at risk of obesity and its metabolic consequences because of a history of obesity in one or both biological parents. Parental obesity was defined as a BMI $>30 \mathrm{~kg} / \mathrm{m}^{2}$ or an elevated waist circumference $(>102 \mathrm{~cm}$ in males and $>88 \mathrm{~cm}$ in females $\left.{ }^{(13)}\right)$. Children with a history of type 1 diabetes or T2D were considered ineligible.

The recruitment process and study design have been extensively described elsewhere ${ }^{(12)}$. Briefly, children in third, fourth and fifth grades from elementary schools located in the proximity of three major urban centres in the province of Quebec (Canada) were approached to participate in the study. Data on 630 families were available for the present study. All the participants underwent the following assessments: blood sample assessment; anthropometric measurements; sexual maturity stage assessment; lifestyle habit assessment using questionnaires; physical activity measurements using accelerometers. The present study was conducted according to the guidelines laid down in the Declaration of Helsinki, and all procedures involving human participants were approved by the Ethics Boards of the Centre Hospitalier Universitaire Sainte-Justine, McGill University and Université Laval. Written informed assent and consent were obtained from all the participants and their parents, respectively.

Dietary intake was assessed using three non-consecutive $24 \mathrm{~h}$ dietary recalls completed by a dietitian with a child (with help from a parent where needed) on different days of the week including one weekend day. To minimise the response burden on families, the recalls were conducted by telephone with each participant using small disposable kits containing food portion models (e.g. graduated cup and bowl), which the participant received before the interview. During the clinical visit, the participants were given a short training session on the use of the kits. Outliers in the analysis of the distribution of each nutrient were examined. Records of intakes with very high or low values of a nutrient were examined for any data entry mistakes (e.g. a whole pie was entered when a slice of pie of a certain dimension was noted as consumed by the child). The recalls were done by dietitians, and children reporting unreasonable intakes were questioned further to ascertain their intake. In the analyses, no high or low values were excluded.

The dietary data were entered into a program that uses the 2007 version of the Canadian Nutrient File. The nutrient analysis program (CANDAT; Godin and Assoc, London, ON) provided daily nutrient intake data, including the participant's total daily intake of carbohydrate, protein, and saturated and unsaturated fat. The calculated average daily percentage of energy from dietary fat, protein and carbohydrate was used, as well as total fibre and energy intake. In terms of foods, measures of sugar-sweetened beverages (SSB, in $\mathrm{ml}$ ) and portions of fruits and vegetables were also calculated. SSB included soft drinks, sports drinks and fruit drinks, but not chocolate milk and $100 \%$ fruit juices.

Physical activity was assessed using an Actigraph LS 7164 activity monitor (Actigraph LLC). Valid wear time was considered to be a minimum of $10 \mathrm{~h} / \mathrm{d}$. Non-wear time was defined as any period of $60 \mathrm{~min}$ or more of zero counts, accepting $1 \mathrm{~min}$ or two consecutive minutes where count values were $>0$ and $\leq 100^{(14)}$. Moderate-to-vigorous physical activity (MVPA) was computed by adding the total minutes spent daily on moderate and vigorous physical activities and averaging over the total number of valid days of wear ${ }^{(15,16)}$. Fitness was estimated using peak $\mathrm{VO}_{2}$, the gold standard for measuring aerobic fitness in youth. $\mathrm{VO}_{2}$ peak was determined during an adapted standard incremental exercise test ${ }^{(17)}$, on an electromagnetic bicycle, to volitional exhaustion with indirect 
calorimetric measurements throughout the test. Attainment of at least one of the following two criteria was required to consider $\mathrm{VO}_{2}$ peak as a true maximum value: (1) a RER $\left(\mathrm{VCO}_{2} / \mathrm{VO}_{2}\right)$ of or greater than 1.0 and (2) a heart rate of or above 185 beats per $\min ^{(18)}$. $\mathrm{VO}_{2}$ peak is expressed in $\mathrm{ml} / \mathrm{kg}$ of lean body mass per-min. Screen time (ST) was assessed using an interviewer-administered questionnaire, documenting self-reported habitual daily hours spent on television viewing and leisure computer/video game use. There were separate questions addressing weekday and weekend ST. From these questions, we calculated the average daily hours of leisure ST.

All the participants underwent a $2 \mathrm{~h}$ oral glucose tolerance test (OGTT) following a $12 \mathrm{~h}$ overnight fast. Blood samples were collected in tubes containing $1 \mathrm{~g} \mathrm{~K}_{2}$ EDTA/1 30, 60, 90 and $120 \mathrm{~min}$ after consumption of an oral glucose dose of $1.75 \mathrm{~g} / \mathrm{kg}$ body weight (to a maximum of $75 \mathrm{~g}$ ). Plasma insulin concentrations were measured using the ultrasensitive Access $^{\circledR}$ immunoassay system (Beckman Coulter, Inc.), which has no cross-reactivity with proinsulin or C-peptide ${ }^{(19)}$. Plasma glucose concentrations were computed on the Beckman Coulter Synchron LX ${ }^{\circledR} 20$ (Beckman Coulter, Inc.) automat using the glucose oxidase method. Analyses were performed in batches at a single site (Centre Hospitalier Universitaire, Sainte-Justine Clinical Biochemistry Laboratory) twice monthly. IFG was defined as a fasting blood glucose value $\geq 5.6 \mathrm{mmol} / 1$ but $<7.0 \mathrm{mmol} / 1$, and IGT was defined as a $2 \mathrm{~h}$ post-load glucose value $\geq 7.8 \mathrm{mmol} / \mathrm{l}$ but $<11.1 \mathrm{mmol} / 1$. Diabetes was defined as a fasting blood glucose value $\geq 7.0 \mathrm{mmol} / \mathrm{l}$ or a $2 \mathrm{~h}$ post-load glucose value $\geq 11.1 \mathrm{mmol} / \mathrm{l}$; at least two such values at different time points, or symptoms consistent with diabetes mellitus, are required to make this diagnosis ${ }^{(20)}$

We used both OGTT-derived indices and fasting-based indices to measure IS and insulin secretion. The fastingbased measure of IS was the homeostasis model assessment of insulin resistance (HOMA-IR), calculated as fasting insulin $(\mathrm{mU} / \mathrm{l}) \times$ fasting glucose $(\mathrm{mmol} / \mathrm{l}) / 22 \cdot 5^{(21)}$. HOMA-IR has been extensively validated against the hyperinsulinaemic-euglycaemic clamp, the gold standard method for assessing IS, in adults and children ${ }^{(22,23)}$. We used the Matsuda IS index (Matsuda ISI) as an OGTT-derived measure of IS. The Matsuda ISI is computed as $10000 / \sqrt{((\text { fasting glucose } \times \text { fasting insulin })}$

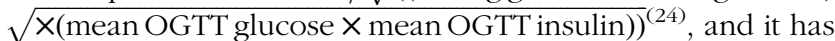
been validated against the current gold standard method of estimating IS in children ${ }^{(22,25)}$. As a measure of fasting insulin secretion, we used HOMA2\%- $\beta$, the updated computer version of the HOMA model. We had previously shown that HOMA $2 \%-\beta$ correlated well with the acute insulin response to glucose derived from a frequently sampled intravenous glucose tolerance test ${ }^{(26)}$ in healthy children. HOMA $2 \% \beta$, as well as the corresponding fasting-based measure of IS (HOMA2-IS), was calculated using the HOMA2 calculator version 2.2 (available at http://www.dtu.ox.ac.uk/homa). OGTT-derived measures of insulin secretion included the ratio of the AUC of insulin:glucose over the first $30 \mathrm{~min}$ (AUC $\mathrm{I} / \mathrm{G}_{t=30 \mathrm{~min}}$ ) of the OGTT (first-phase insulin secretion) and the AUC of insulin:glucose over the complete $2 \mathrm{~h}$ (AUC $\mathrm{I} / \mathrm{G}_{t=120 \mathrm{~min}}$ ) of the OGTT (second-phase insulin secretion). The AUC $\mathrm{I} / \mathrm{G}_{t=30 \mathrm{~min}}$ has previously been shown to have a good correlation with the acute insulin response to glucose in healthy children $^{(26)}$. Body composition was determined using dualenergy X-ray absorptiometry (Prodigy Bone Densitometer System, DF+14664; GE Lunar Corporation) ${ }^{(27)}$. The percentage of total body fat mass was used as the measure of adiposity. Pubertal development, based on Tanner stages, was assessed by the inspection of genitalia and pubic hair development in boys and breast and pubic hair development in girls by a trained nurse ${ }^{(28,29)}$. Children were then categorised as pre-pubertal (Tanner stage 1) or pubertal (Tanner stages 2-5).

\section{Statistical analyses}

Descriptive statistics, including means and standard deviations, as well as proportions, were used to describe the baseline characteristics of the participants and their dietary intake of major macronutrients. Measures were analysed as continuous variables where possible. Age- and sex-adjusted BMI $z$-scores were computed from Centers for Disease Control and Prevention (2000) data ${ }^{(30)}$. A BMI $z$-score $<5$ th percentile for age and sex defined underweight, a BMI $z$-score $\geq 5$ th percentile but $<85$ th percentile for age and sex defined normal weight, and a BMI $z$-score $\geq 85$ th percentile but $<95$ th percentile for age and sex defined overweight, while a BMI $z$ score $\geq 95$ th percentile for age and sex defined obesity. Univariable and multivariable linear regressions were used to examine the associations between macronutrient intake and measures of insulin secretion and IS (outcome variables). All multivariable models were adjusted for physical activity, fitness, sedentary behaviour and adiposity, as well as sex, age and stage of pubertal development. We assessed the linearity of the associations using a test of non-linearity ${ }^{(31)}$ and modelled all non-linear associations using non-parametric smoothing splines with four degrees of freedom, in which the shape of the association was estimated directly from the data, using generalised additive models ${ }^{(31)}$. For parsimony, and given that physical activity is reduced primarily in the winter months ${ }^{(32)}$, we defined two periods: 'winter', from November to March, and 'non-winter', from April to October. We included season in models that included physical activity. Outcome variables had non-symmetric distributions and were log-transformed using $100 \times \ln$ of the variable, such that $\beta$-coefficients represent the percentage change in the outcome associated with a one-unit increase in the exposure variable ${ }^{(33)}$. Interaction terms were introduced one at a time into the models to examine whether the association between physical activity and IS, between fitness and IS, or between ST and IS varied as a function of sex. All analyses were performed using Statistical Analysis Systems statistical software package version 9.2 (SAS Institute, Inc.).

\section{Results}

Of the 630 participants in the QUALITY cohort, 215 participants were excluded due to missing dietary data or not having adequate accelerometer or fitness test data (based on 
our predefined criteria of a minimum accelerometer wear time of $10 \mathrm{~h} / \mathrm{d}$ for a minimum of $4 \mathrm{~d}$ and a maximal fitness test value). There were no statistically significant differences between these participants and those included in the analyses in terms of sex and BMI $z$-scores (data not shown). The excluded participants were slightly younger (mean age $9.3 \mathrm{v}$. 9.7 years) than those included in the analyses $(P<0.0001)$. We had found previously that participants with a sub-maximal fitness test value were younger than those with a maximal fitness test value, probably due to a greater difficulty in younger children to meet the strenuous demands of a cycling test, such as pedalling against an increasing workload while following an imposed rhythm ${ }^{(34)}$. The selected characteristics of the 415 participants retained for the analyses are presented in Table 1. Of the participants, $55 \%$ were of normal weight for their age and sex, 20\% were overweight and 23\% were obese, with $2 \%$ being underweight. Table 2 outlines the nutrient intake data by sex across participants of the QUALITY cohort. Overall, boys consumed more energy daily as well as higher quantities of dietary vitamin $\mathrm{D}$ and fibre than girls. Girls tended to drink less SSB than boys $(P=0 \cdot 087)$.

In our sample, fifteen children had IFG, thirty-one youth had IGT and five children had both IFG and IGT. Although four children met the definition of diabetes based on either their fasting or $2 \mathrm{~h}$ post-load glucose values, at least two such values at different time points, or symptoms consistent with diabetes mellitus, are required to make this diagnosis.

When examining the univariable association between macronutrient intake and indices of IS, only protein and fibre intakes had significant associations. For every $1 \%$ increase in daily protein intake, HOMA-IR increased by almost $2 \%$. Conversely, for every additional gram of fibre consumed, HOMA-IR decreased by $1.66 \%$ (Table 3). However, these associations were no longer significant when adjusting for other lifestyle habits, such as physical activity and ST, as well as adiposity. Similarly, for every additional gram of fibre consumed daily, the Matsuda ISI increased by $1.5 \%$. There was a similar trend of decreased IS measured by the Matsuda ISI with higher protein intake $(P=0 \cdot 051)$. No association between dietary macronutrient intake and the Matsuda ISI remained significant when accounting for MVPA, fitness, ST, adiposity and other covariates (Table 3).

The percentage of protein also appeared to be associated with first-phase insulin secretion: for every $1 \%$ increase in daily protein intake, first-phase insulin secretion decreased by $1 \cdot 1 \%$, after controlling for MVPA, fitness, ST, adiposity and other covariates (data not shown). This association, however, did not hold true for HOMA $2 \%-\beta$ or second-phase insulin secretion. Moreover, although higher intakes of SSB appeared to be associated with lower second-phase insulin

Table 1. Baseline characteristics of the participants aged 8-10 years, according to sex (QUebec Adipose and Lifestyle InvesTigation in Youth cohort)

(Mean values and standard deviations; number of participants and percentages; medians and ranges)

\begin{tabular}{|c|c|c|c|c|c|}
\hline & \multicolumn{2}{|c|}{ Boys ( $n$ 218) } & \multicolumn{2}{|c|}{ Girls (n 197) } & \multirow[b]{2}{*}{$P^{*}$} \\
\hline & $n$ & $\%$ & $n$ & $\%$ & \\
\hline Age (years) & & \multirow{2}{*}{\multicolumn{2}{|c|}{$9 \cdot 7$}} & 0.523 \\
\hline Mean & \multirow{2}{*}{\multicolumn{2}{|c|}{$\begin{array}{l}9.7 \\
0.9\end{array}$}} & & & \\
\hline SD & & & \multicolumn{2}{|c|}{0.9} & \\
\hline BMI $z$-score & \multicolumn{2}{|c|}{0.9} & & & 0.436 \\
\hline Mean & \multicolumn{2}{|c|}{0.7} & \multicolumn{2}{|c|}{0.8} & \\
\hline Range & \multicolumn{2}{|c|}{$-1 \cdot 8,2 \cdot 6$} & \multicolumn{2}{|c|}{$-3 \cdot 2,2 \cdot 6$} & \\
\hline \multicolumn{5}{|l|}{ BMI category $\left(\mathrm{kg} / \mathrm{m}^{2}\right)$} & 0.467 \\
\hline Underweight & 4 & $1 \cdot 8$ & 4 & $2 \cdot 0$ & \\
\hline Normal weight & 124 & $56 \cdot 9$ & 106 & 53.8 & \\
\hline Overweight & 46 & $21 \cdot 1$ & 35 & $17 \cdot 8$ & \\
\hline Obese & 44 & $20 \cdot 2$ & 52 & $26 \cdot 4$ & \\
\hline \multicolumn{5}{|l|}{ Tanner stage } & $<0.0001$ \\
\hline Pre-pubertal & 195 & 89.5 & 121 & $61 \cdot 4$ & \\
\hline Pubertal & 23 & $10 \cdot 6$ & 76 & 38.6 & \\
\hline \multicolumn{5}{|l|}{ Percentage of fat mass } & $<0.0001$ \\
\hline Mean & \multicolumn{2}{|c|}{$23 \cdot 6$} & \multicolumn{2}{|c|}{$30 \cdot 2$} & \\
\hline SD & \multicolumn{2}{|c|}{$10 \cdot 9$} & \multicolumn{2}{|c|}{$10 \cdot 2$} & \\
\hline MVPA (min/d) & & & & & $<0.0001$ \\
\hline Mean & \multicolumn{2}{|c|}{58.4} & \multicolumn{2}{|c|}{$40 \cdot 8$} & \\
\hline SD & \multicolumn{2}{|c|}{$26 \cdot 7$} & \multicolumn{2}{|c|}{$18 \cdot 8$} & \\
\hline Screen time $(h / d)$ & \multirow{2}{*}{\multicolumn{2}{|c|}{$2 \cdot 6$}} & & & $<0.0001$ \\
\hline Median & & & \multicolumn{2}{|c|}{1.8} & \\
\hline Range & \multicolumn{2}{|c|}{$0.1-13 \cdot 4$} & \multicolumn{2}{|c|}{$0.03-10.1$} & \\
\hline $\mathrm{VO}_{2}$ peak $(\mathrm{ml} / \mathrm{kg} \mathrm{LBM}$ per $\mathrm{min})$ & \multirow{2}{*}{\multicolumn{2}{|c|}{$60 \cdot 6$}} & \multirow{2}{*}{\multicolumn{2}{|c|}{57.4}} & $<0.0001$ \\
\hline Mean & & & & & \\
\hline SD & \multicolumn{2}{|c|}{5.9} & \multicolumn{2}{|c|}{$6 \cdot 0$} & \\
\hline
\end{tabular}

MVPA, moderate-to-vigorous physical activity; LBM, lean body mass.

* The $P$ value indicates the statistical significance of a $t$ test comparing mean values between boys and girls for normally distributed variables or a Mann-Whitney $U$ test for variables with a skewed distribution. $\chi^{2}$ test or Fisher's exact test was used for categorical variables. 
Table 2. Baseline nutrient intake data by sex for the participants of QUebec Adipose and Lifestyle InvesTigation in Youth

(Mean values and standard deviations; medians and ranges)

\begin{tabular}{|c|c|c|c|c|c|}
\hline \multirow[b]{2}{*}{ Components } & \multicolumn{2}{|c|}{ Boys ( $n 218)$} & \multicolumn{2}{|c|}{ Girls $(n$ 197) } & \multirow[b]{2}{*}{$P^{*}$} \\
\hline & Mean & SD & Mean & SD & \\
\hline Total energy (kcal) & 1755 & 414 & 1571 & 367 & $<0.0001$ \\
\hline Total energy (kJ) & 7343 & 1732 & 6573 & 1536 & $<0.0001$ \\
\hline Percentage of total fat & $32 \cdot 3$ & 4.9 & $32 \cdot 4$ & 4.7 & 0.722 \\
\hline Percentage of saturated fat & 11.4 & 2.5 & 11.6 & $2 \cdot 6$ & 0.442 \\
\hline Percentage of carbohydrate & $52 \cdot 8$ & $6 \cdot 3$ & $53 \cdot 0$ & $6 \cdot 7$ & 0.725 \\
\hline Percentage of protein & $16 \cdot 4$ & 3.4 & $16 \cdot 0$ & 3.5 & 0.224 \\
\hline Vitamin D $(\mu \mathrm{g} / \mathrm{d})$ & $6 \cdot 2$ & $4 \cdot 1$ & $5 \cdot 0$ & $3 \cdot 0$ & 0.004 \\
\hline Portions of fruits and vegetables & 4.5 & $2 \cdot 1$ & $4 \cdot 2$ & $2 \cdot 1$ & 0.187 \\
\hline $\mathrm{SSB}(\mathrm{ml} / \mathrm{d})$ & & & & & 0.087 \\
\hline Median & \multirow{2}{*}{\multicolumn{2}{|c|}{$\begin{array}{c}83 \cdot 2 \\
0-774.9\end{array}$}} & \multirow{2}{*}{\multicolumn{2}{|c|}{$\begin{array}{c}66.7 \\
0-582.2\end{array}$}} & \\
\hline Range & & & & & \\
\hline Fibre $(g / d)$ & $13 \cdot 7$ & 4.4 & 12.5 & 4.0 & 0.003 \\
\hline
\end{tabular}

SSB, sugar-sweetened beverages.

* The $P$ value indicates the statistical significance of a $t$ test comparing mean values between boys and girls for normally distributed variables or a Mann-Whitney $U$ test for variables with a skewed distribution.

secretion $(\beta=-0.02 ; \quad P=0.030)$, this association did not hold when controlling for potential confounders (data not shown). The intake of no other macronutrients, or total daily energy intake, was associated with insulin secretion (across all indices).

\section{Discussion}

In this cohort of youth with a family history of obesity children with higher protein content in their diet appeared to have lower first-phase insulin secretion requirements, even after controlling for other lifestyle habits and adiposity. Otherwise, the intake of no other dietary macronutrient was associated with the measures of IS or insulin secretion after adjusting for MVPA, fitness, ST and adiposity.

Studies examining the association between protein intake and insulin dynamics in youth are limited and have reported conflicting results. Hoppe et al. ${ }^{(8)}$ studied the effect of the intake of animal protein in the form of milk or meat on insulin dynamics in twenty-four 8-year-old Caucasian boys. They found that milk was associated with decreased IS and increased $\beta$-cell function, while the addition of meat to the diet led to unchanged IS and increased $\beta$-cell function. Comparison of the present results with those reported by Hoppe et $a l^{(8)}$ remains difficult, given the different study designs and the fact that their study examined very high protein

Table 3. Association between dietary components and measures of insulin sensitivity* ( $\beta$-Coefficients and standard errors)

\begin{tabular}{|c|c|c|c|c|c|c|}
\hline \multirow[b]{2}{*}{ Components } & \multicolumn{3}{|c|}{ HOMA-IR ( $n$ 413) } & \multicolumn{3}{|c|}{ Adjusted† (n 413) } \\
\hline & $\beta$ & SE & $P$ & $\beta$ & SE & $P$ \\
\hline Total energy $(100 \mathrm{kcal} / \mathrm{d})$ & -0.97 & 0.74 & 0.193 & 0.35 & 0.56 & 0.526 \\
\hline Total energy $(418.4 \mathrm{~kJ} / \mathrm{d})$ & -0.97 & 0.74 & 0.193 & 0.35 & 0.56 & 0.526 \\
\hline Percentage of fat & $0 \cdot 28$ & 0.63 & 0.654 & 0.28 & 0.46 & 0.550 \\
\hline Percentage of saturated fat & -0.12 & $1 \cdot 18$ & 0.919 & 0.13 & 0.86 & 0.883 \\
\hline Percentage of carbohydrate & -0.85 & 0.46 & 0.065 & -0.39 & 0.34 & 0.259 \\
\hline Percentage of protein & 1.96 & 0.86 & 0.024 & 0.60 & 0.65 & 0.355 \\
\hline \multirow[t]{3}{*}{ Fibre $(g / d)$} & $-1 \cdot 66$ & $0 \cdot 70$ & 0.018 & -0.43 & 0.52 & 0.406 \\
\hline & \multicolumn{3}{|c|}{ Matsuda ISI (n 390) } & \multicolumn{3}{|c|}{ Adjusted† (n 390) } \\
\hline & $\beta$ & SE & $P$ & $\beta$ & SE & $P$ \\
\hline Total energy $(100 \mathrm{kcal} / \mathrm{d})$ & $1 \cdot 2$ & 0.8 & 0.103 & -0.18 & 0.54 & 0.737 \\
\hline Total energy $(418.4 \mathrm{~kJ} / \mathrm{d})$ & 1.2 & 0.8 & 0.103 & -0.18 & 0.54 & 0.737 \\
\hline Percentage of fat & 0.08 & 0.62 & 0.901 & 0.04 & 0.44 & 0.926 \\
\hline Percentage of saturated fat & 0.7 & $1 \cdot 2$ & 0.568 & 0.43 & 0.82 & 0.597 \\
\hline Percentage of carbohydrate & 0.5 & 0.46 & 0.252 & 0.08 & 0.32 & 0.815 \\
\hline Percentage of protein & -1.7 & 0.86 & 0.051 & -0.34 & 0.61 & 0.574 \\
\hline Fibre (g/d) & 1.5 & 0.73 & 0.038 & $0 \cdot 11$ & 0.51 & 0.828 \\
\hline
\end{tabular}

HOMA-IR, homeostasis model assessment of insulin resistance; Matsuda ISI, Matsuda insulin sensitivity index.

*Models on the left are univariable linear regression models and models on the right are multivariable models. For HOMA-IR, splines were used to model the association between adiposity and screen time, while only adiposity was modelled using splines for Matsuda ISI.

† Models adjusted for moderate-to-vigorous physical activity, fitness, screen time, adiposity, age, sex, Tanner stage and season. 
intakes. In contrast, a study of 365 school-aged children (age: 10 (SD 2.3) years) from poor suburbs of Buenos Aires examined the association of milk consumption, lifestyle and insulin resistance cross-sectionally ${ }^{(9)}$, and found an inverse association between milk consumption and insulin resistance (measured by HOMA-IR) after adjusting for blocks walked daily, television viewing, soft drink consumption, parental education level, sex, age, HDL-cholesterol levels and systolic blood pressure ${ }^{(9)}$. This study did not consider protein intake from other sources, making comparisons of its results with those of the present study difficult.

On the whole, there are limited data regarding the association of dietary protein intake with insulin dynamics in children, and while it has been suggested - at least acutely - that high dietary protein intakes from dairy products may be more deleterious to IS than meat protein intakes, epidemiological data suggest that exposure to dairy products is beneficial for IS. Further studies are required to examine the influence of dietary protein and its various sources (dairy products $v$. meat) on insulin dynamics.

Dietary intake is critical for the development of obesity: an imbalance between dietary intake and energy expenditure leads to increases in adiposity. We sought to determine whether dietary intake, independently of its established effect on adiposity, is associated with IS and insulin secretion. The low variability of macronutrient intakes across youth in the present study might explain the overall absence of an association between dietary composition and insulin dynamics in youth. Alternatively, the absence of an association may be related to metabolic plasticity in this age group. Indeed, Sunehag et al. ${ }^{(10)}$ examined the impact of adopting various diets in a small group ( $n$ 36) of healthy, non-obese pre-pubertal and adolescent youth using a randomised cross-over design. Specifically, they examined the effect of a high-fat/low-carbohydrate diet and three different high-carbohydrate/low-fat diets: one with low fructose content (where fructose content was $10 \%$ of daily carbohydrate intake); another with moderate fructose content (where fructose represented $20 \%$ of daily carbohydrate intake); the last one with high fructose content (where fructose accounted for $40 \%$ of daily carbohydrate intake). The results suggested that the children adapted rapidly to large changes in carbohydrate and fat intakes by adjusting carbohydrate or fat oxidation, respectively. IS was not different across diets in pre-pubertal children, but was lower in adolescents on the high-fat diet, compared with those on the high-carbohydrate diet. Furthermore, first- and second-phase insulin secretion remained similar across diets, regardless of age and sex ${ }^{(29)}$. Similarly, in these children, high-carbohydrate diets were not associated with any changes in IS in the pre-pubertal youth, while they were associated with improved IS in the adolescent group. Moreover, pancreatic $\beta$-cell function was not adversely affected by such highcarbohydrate diets: indices of first- and second-phase insulin secretion remained unchanged among the pubertal and prepubertal youth ${ }^{(10)}$. It may be that dietary composition has little effect on insulin and glucose dynamics early in life, but that its relevance becomes more important with age. Adolescents in Sunehag's study tended to exhibit changes in IS based on dietary composition, suggesting that changes may occur in adolescence, but not in pre-pubertal youth. Longitudinal studies are required to determine whether dietary macronutrient composition exerts an effect on IS and insulin secretion independently of its effects on adiposity.

The findings of the present study are complementary to those of our previous work examining how SSB consumption is associated with the components of the metabolic syndrome ${ }^{(35)}$. In our previous work, we found that among overweight children ( $\geq 85$ th BMI percentile), a $100 \mathrm{ml}$ increase in SSB consumption was associated with $0 \cdot 10$ unit higher HOMA-IR, after controlling for age, sex, adiposity and overall physical activity (measured in counts per min). In the present study, we furthered our understanding of the association of lifestyle habits with metabolic health by taking into consideration additional or more specific aspects of lifestyle habits. Specifically, we adjusted for MVPA, which has been reported to be more closely associated with metabolic health than with overall physical activity. Furthermore, we adjusted for fitness, which reflects the body's potential for substrate utilisation. Finally, we also adjusted for sedentary behaviour, recently found to have an independent association with metabolic health by our group ${ }^{(34)}$. Considering these lifestyle factors jointly suggest that fitness, and lifestyle habits other than dietary habits, may be more important to metabolic health in children.

The strengths of the present study lie in its relatively large sample size and the high quality of measures obtained, specifically physical activity, fitness, adiposity and dietary composition. The use of three consecutive $24 \mathrm{~h}$ dietary recalls optimises data collection on habitual dietary habits. This method has been validated using the gold standard of doubly labelled water ${ }^{(36)}$. In addition, the telephone interview method has been used in several settings with good results in adults ${ }^{(37)}$ and children ${ }^{(38)}$. Furthermore, we attempted to adjust for other lifestyle factors, such as fitness, physical activity and sedentary behaviour, for determining the association between dietary macronutrient content and insulin dynamics. The limitations of the present study include its cross-sectional nature, which prevents causal inference. Also, the findings of the present study are generalisable only to Caucasian youth with a parental history of obesity; however, this group comprises a large segment of the Canadian population and constitutes a relevant 'at-risk' group. While there are no population-based prevalence rates for children with IGT, population-based prevalence rates for IFG tend to be those of older adolescents and various at-risk ethnic groups, making comparisons with our sample difficult. Based on the findings of the present study, we report prevalence rates of $3.6 \%$ for children with IFG alone, $1.2 \%$ for those with both IFG and IGT, and $7.5 \%$ for those with IGT alone, rates which are in keeping with a recent study that has examined the prevalence rates of IFG in overweight and obese 6-10-year-olds ${ }^{(39)}$.

In conclusion, our findings suggest that in children with a parental history of obesity, higher protein intake is associated with lower first-phase insulin secretion requirements. Overall, while long-term excess of energy has been shown to lead to 
overweight and obesity, dietary macronutrient composition does not appear to be directly correlated with IS and insulin secretion. This may be due to metabolic plasticity in youth and suggests that strategies to prevent T2D mellitus in youth should focus on lifestyle modifications other than dietary composition (e.g. MVPA).

\section{Acknowledgements}

The authors thank Dr Marie-Ève Mathieu (Université de Montréal) for her editorial support. Dr Marie Lambert (July 1952-February 2012), paediatric geneticist and researcher, initiated the QUALITY cohort. Her leadership and devotion to QUALITY will always be remembered and appreciated. They are also grateful to all the families that participated in QUALITY.

QUALITY is funded by the Canadian Institutes of Health Research, the Heart and Stroke Foundation of Canada and the Fonds de la recherche en santé du Québec. The Canadian Institutes of Health Research, the Heart and Stroke Foundation of Canada and the Fonds de la recherche en santé du Québec had no role in the design and analyses of the study or writing of this article.

The authors' contributions are as follows: M. H. researched the data, performed the analyses and wrote the manuscript; K. G.-D. researched the data, contributed to the discussion and reviewed/edited the manuscript; A. B. assisted with the analyses and reviewed/edited the manuscript.

The authors have no conflicts of interest to disclose.

\section{References}

1. Shields M (2006) Overweight and obesity among children and youth. Health Rep 17, 27-42.

2. Li C, Ford ES, Zhao G, et al. (2009) Prevalence of pre-diabetes and its association with clustering of cardiometabolic risk factors and hyperinsulinemia among U.S. adolescents: National Health and Nutrition Examination Survey 2005-2006. Diabetes Care 32, 342-347.

3. Sinha R, Fisch G, Teague B, et al. (2002) Prevalence of impaired glucose tolerance among children and adolescents with marked obesity. $N$ Engl J Med 346, 802-810.

4. Lambert M, Delvin EE, Levy E, et al. (2008) Prevalence of cardiometabolic risk factors by weight status in a population-based sample of Quebec children and adolescents. Can J Cardiol 24, 575-583.

5. Knowler WC, Barrett-Connor E, Fowler SE, et al. (2002) Reduction in the incidence of type 2 diabetes with lifestyle intervention or metformin. N Engl J Med 346, 393-403.

6. Lindquist CH, Gower BA \& Goran MI (2000) Role of dietary factors in ethnic differences in early risk of cardiovascular disease and type 2 diabetes. Am J Clin Nutr 71, 725-732.

7. Arslanian SA, Saad R, Lewy V, et al. (2002) Hyperinsulinemia in African-American children: decreased insulin clearance and increased insulin secretion and its relationship to insulin sensitivity. Diabetes 51, 3014-3019.

8. Hoppe C, Molgaard C, Vaag A, et al. (2005) High intakes of milk, but not meat, increase s-insulin and insulin resistance in 8-year-old boys. Eur J Clin Nutr 59, 393-398.

9. Hirschler V, Oestreicher K, Beccaria M, et al. (2009) Inverse association between insulin resistance and frequency of milk consumption in low-income Argentinean school children. J Pediatr 154, 101-105.
10. Sunehag AL, Toffolo G, Treuth MS, et al. (2002) Effects of dietary macronutrient content on glucose metabolism in children. J Clin Endocrinol Metab 87, 5168-5178.

11. Davis JN, Ventura EE, Shaibi GQ, et al. (2007) Reduction in added sugar intake and improvement in insulin secretion in overweight Latina adolescents. Metab Syndr Relat Disord 5, 183-193.

12. Lambert M, Van Hulst A, O'Loughlin J, et al. (2011) Cohort profile: the Quebec adipose and lifestyle investigation in youth cohort. Int J Epidemiol 41, 1533-1544.

13. Grundy SM, Cleeman JI, Daniels SR, et al. (2005) Diagnosis and management of the metabolic syndrome: an American Heart Association/National Heart, Lung, and Blood Institute Scientific Statement. Circulation 112, 2735-2752.

14. Troiano RP, Berrigan D, Dodd KW, et al. (2008) Physical activity in the United States measured by accelerometer. Med Sci Sports Exerc 40, 181-188.

15. Evenson KR, Catellier DJ, Gill K, et al. (2008) Calibration of two objective measures of physical activity for children. J Sports Sci 26, 1557-1565.

16. Trost SG, Loprinzi PD, Moore R, et al. (2011) Comparison of accelerometer cut points for predicting activity intensity in youth. Med Sci Sports Exerc 43, 1360-1368.

17. Skinner JS (editor) (1993) Exercise Testing and Exercise Prescription for Special Cases: Theoretical Basis and Clinical Application, 3rd ed, p. 67. Baltimore, MD: Lippincott Williams \& Wilkins.

18. Docherty D (editor) (1996) Measurement in Pediatric Exercise Science. Champaign, IL: Human Kinetics Publishers.

19. Allard P, Delvin EE, Paradis G, et al. (2003) Distribution of fasting plasma insulin, free fatty acids, and glucose concentrations and of homeostasis model assessment of insulin resistance in a representative sample of Quebec children and adolescents. Clin Chem 49, 644-649.

20. Canadian Diabetes Association Clinical Practice Guidelines Expert Committee (2008) Canadian Diabetes Association Clinical Practice Guidelines for the prevention and management of diabetes in Canada. Can J Diabetes 32, S10-S13.

21. Matthews DR, Hosker JP, Rudenski AS, et al. (1985) Homeostasis model assessment: insulin resistance and beta-cell function from fasting plasma glucose and insulin concentrations in man. Diabetologia 28, 412-419.

22. Henderson M, Rabasa-Lhoret R, Bastard JP, et al. (2011) Measuring insulin sensitivity in youth: how do the different indices compare with the gold-standard method? Diabetes Metab 37, 72-78.

23. Gungor N, Saad R, Janosky J, et al. (2004) Validation of surrogate estimates of insulin sensitivity and insulin secretion in children and adolescents. J Pediatr 144, 47-55.

24. Matsuda M \& DeFronzo RA (1999) Insulin sensitivity indices obtained from oral glucose tolerance testing: comparison with the euglycemic insulin clamp. Diabetes Care 22, $1462-1470$.

25. Yeckel CW, Weiss R, Dziura J, et al. (2004) Validation of insulin sensitivity indices from oral glucose tolerance test parameters in obese children and adolescents. J Clin Endocrinol Metab 89, 1096-1101.

26. Henderson M, Baillargeon JP, Rabasa-Lhoret R, et al. (2012) Estimating insulin secretion in youth using simple indices derived from the oral glucose tolerance test. Diabetes Metab 38, 309-315.

27. Goran MI (1998) Measurement issues related to studies of childhood obesity: assessment of body composition, body fat distribution, physical activity, and food intake. Pediatrics 101, 505-518. 
28. Marshall WA \& Tanner JM (1969) Variations in pattern of pubertal changes in girls. Arch Dis Child 44, 291-303.

29. Marshall WA \& Tanner JM (1970) Variations in the pattern of pubertal changes in boys. Arch Dis Child 45, 13-23.

30. Centers for Disease Control and Prevention (2000) Growth Charts. http://cdc.gov/growthcharts/ (accessed 12 August 2013).

31. Hastie T \& Tibshirani R (1990) Generalized Additive Models. New York, NY: Chapman and Hall.

32. Belanger M, Gray-Donald K, O'Loughlin J, et al. (2009) Influence of weather conditions and season on physical activity in adolescents. Ann Epidemiol 19, 180-186.

33. Cole TJ (2000) Sympercents: symmetric percentage differences on the $100 \log (e)$ scale simplify the presentation of $\log$ transformed data. Stat Med 19, 3109-3125.

34. Henderson M, Gray-Donald K, Mathieu ME, et al. (2012) How are physical activity, fitness, and sedentary behavior associated with insulin sensitivity in children? Diabetes Care 35, 1272-1278.
35. Wang JW, Mark S, Henderson M, et al. (2012) Adiposity and glucose intolerance exacerbate components of metabolic syndrome in children consuming sugar-sweetened beverages: QUALITY cohort study. Pediatr Obes 8, 284-293.

36. Burrows TL, Martin RJ \& Collins CE (2010) A systematic review of the validity of dietary assessment methods in children when compared with the method of doubly labeled water. J Am Diet Assoc 110, 1501-1510.

37. Conway JM, Ingwersen LA \& Moshfegh AJ (2004) Accuracy of dietary recall using the USDA five-step multiple-pass method in men: an observational validation study. $J \mathrm{Am}$ Diet Assoc 104, 595-603.

38. Baxter SD, Thompson WO, Litaker MS, et al. (2003) Accuracy of fourth-graders' dietary recalls of school breakfast and school lunch validated with observations: in-person versus telephone interviews. J Nutr Educ Behav 35, 124-134.

39. Moadab MH, Kelishadi R, Hashemipour M, et al. (2010) The prevalence of impaired fasting glucose and type 2 diabetes in a population-based sample of overweight/obese children in the Middle East. Pediatr Diabetes 11, 101-106. 